\title{
Foreword on the special issue: from discrete particles to continuum models of granular mechanics
}

\author{
Ken Kamrin ${ }^{1}$
}

Published online: 25 November 2016

(C) OWZ 2016

The mechanics of granular materials have remained a challenge to describe since the days of Coulomb, both theoretically and computationally. Granular media arise in a number of everyday circumstances (e.g., raw materials, soils, food/agriculture, pharmaceuticals, vehicle mobility, debris removal) and remain the second-most handled material by weight in global industry. Hence, the challenge of coming up with accurate and efficient ways to simulate a collection of grains is an issue of great import to multiple disciplines. There are two major perspectives. Ideally, one would like a continuum model able to describe the behavior of large collections of grains in a numerically efficient fashion. There is also the discrete perspective, where modeling arises at the individual particle level and the motion of every grain is tracked and evolved under mechanical laws.

To calculate large-scale motion and grain statistics at the continuum level, complexities arise in determining and solving upscaled models, such as (i) the presence of size effects due to the finite size of grains, which points to a nonlocal continuum approach; (ii) the tendency for grains to segregate based on grain size during flow, and the corresponding need for robust homogenization methods to extract continuum fields in the presence of varying polydispersity; and (iii) nontrivial kinetic heat transfer and energy loss from dissipative/frictional particle collisions, especially as the material packing goes from dilute to close-packed. Challenges also arise in the discrete particle perspective. The case of spherical, stiff grains may be well described with frictional visco-elastic contacts based on Hertzian interactions, however, real systems often invoke complex angular grain shapes and are made of materials with diverse mechanical behaviors. In these cases, the grain interactions can go beyond cohesionless contact, the geometry of contact goes beyond that of two smooth surfaces, and damage or fracture laws may be needed to describe the internal grain mechanics.

To address the challenges above, a combination of advanced modeling and novel computational approaches is needed. The seven papers included in this special issue cover the range from continuum methodology to higher-order discrete approaches, to address many of the open challenges described above. The topics have been selected based on presentations in the symposium, "From Discrete Particles to Continuum Models of Granular Mechanics," held at the Fourth International Conference on Particle-Based Methods in Barcelona, 2015.

For questions please contact Ken Kamrin at kkamrin@mit.edu, or the individual corresponding authors.
Ken Kamrin

kkamrin@mit.edu

1 Department of Mechanical Engineering, MIT, Cambridge, MA 02139, USA 